\title{
Individuality in FGFI expression significantly influences platinum resistance and progression-free survival in ovarian cancer
}

\author{
G Smith ${ }^{*, 1}$, MTH Ng ${ }^{1,5}$, L Shepherd ${ }^{1,5}$, CS Herrington', C Gourley ${ }^{2}$, MJ Ferguson ${ }^{3}$ and CR Wolf',4 \\ 'Division of Cancer Research, Medical Research Institute, University of Dundee, Dundee, UK; '² Einburgh Cancer Research Centre, MRC IGMM, University \\ of Edinburgh, Western General Hospital, Edinburgh EH2 2XR, UK; ${ }^{3}$ Tayside Cancer Centre, Ninewells Hospital and Medical School, Dundee DDI 9SY, UK; \\ ${ }^{4}$ CRUK Molecular Pharmacology Unit, Ninewells Hospital and Medical School, Dundee DDI 9SY, UK
}

BACKGROUND: Ovarian cancer is frequently advanced at presentation when treatment is rarely curative. Response to first-line platinum-based chemotherapy significantly influences survival, but clinical response is unpredictable and is frequently limited by the development of drug-resistant disease.

METHODS: We used qRT-PCR analysis to assess intertumour differences in the expression of fibroblast growth factor I (FGFI) and additional candidate genes in human ovarian tumours $(n=187)$, and correlated individuality in gene expression with tumour histology, chemotherapy response and survival. We used MTT assays to assess platinum chemosensitivity in drug-sensitive and drug-resistant ovarian cell lines.

RESULTS: Marked intertumour differences in gene expression were observed, with each tumour having a unique gene expression profile. Nine genes, including FGFI $\left(P=1.7 \times 10^{-5}\right)$ and FGFR2 $(P=0.003)$, were differentially expressed in serous and nonserous tumours. MDM2 $(P=0.032)$ and ERBB2 $(P=0.064)$ expression was increased in platinum-sensitive patients, and FGFI (adjusted logrank test $P=0.006)$, FGFR2 $(P=0.04)$ and PDRFRB expression $(P=0.037)$ significantly inversely influenced progression-free survival. Stable FGFI gene knockdown in platinum-resistant A2780DPP cells re-sensitised cells to both cisplatin and carboplatin.

CONCLUSION: We show for the first time that FGFI is differentially expressed in high-grade serous ovarian tumours, and that individuality in FGFI expression significantly influences progression-free survival and response to platinum-based chemotherapy. British Journal of Cancer (2012) 107, 1327-1336. doi:10.1038/bjc.2012.410 www.bjcancer.com

Published online 18 September 2012

(C) 2012 Cancer Research UK

Keywords: fibroblast growth factors; ovarian cancer; survival; chemotherapy; platinum drugs

Epithelial ovarian cancer, the second most common and most lethal gynaecological malignancy, frequently presents at an advanced stage where surgery is difficult and metastatic spread common (http://www.isdscotlandarchive.scot.nhs.uk/isd/Cancer_ in_Scotland_summary.pdf). Treatment is therefore rarely curative, with 5-year survival $<10 \%$ in patients with stage IV disease (Kristensen and Trope, 1997). Standard treatment combines cytoreductive surgery and adjuvant or neoadjuvant chemotherapy with platinum-taxane drug regimens (Scottish Intercollegiate Guidelines Network, 2003), but prolonged response is frequently compromised by the development of multidrug-resistant disease (Clark et al, 2001).

Ovarian cancer (ICD-10 C56) describes several related diseases with different cellular origins and molecular characteristics (Kobel et al, 2008). The majority of high-grade serous cancers, the most common and aggressive histological subtype, originate in the distal fallopian tube (Lee et al, 2007) and show strong nuclear expression of the Wilms' tumour (WT-1) protein (Al-Hussaini et al, 2004). Clear-cell cancers, in contrast, show similarities to renal cancers (Zorn et al, 2005), whereas endometrioid cancers show similar

\footnotetext{
*Correspondence: Dr G Smith; E-mail: g.smith@dundee.ac.uk

${ }^{5}$ These authors contributed equally to this work.

Received 4 July 20 I2; revised I4 August 2012; accepted I4 August 2012; published online 18 September 2012
}

molecular abnormalities to endometriosis (Wiegand et al, 2010), and mucinous ovarian cancers may represent metastases from primary tumours in the gastrointestinal tract (Lee and Young, 2003). Despite the molecular diversity of the different histological subtypes, ovarian cancer continues to be largely treated as a single disease, in accordance with the national guidelines (Scottish Intercollegiate Guidelines Network, 2003), and there are no validated patient selection or chemotherapy response biomarkers in routine clinical use.

Recent approaches to chemotherapy drug design have focussed on inhibitors of angiogenesis, the process of new blood vessel formation that promotes cancer progression, associated with ovarian tumour growth, metastatic spread and ascites formation (Brown et al, 2000). Angiogenesis inhibitors are primarily targeted to growth factors (e.g., EGF, FGF, PDGF and VEGF) and associated receptor tyrosine kinases (RTKs) that regulate key signal transduction pathways frequently dysregulated in ovarian cancer (Burger, 2011). VEGF ligands, for example, activate signalling pathways including RAS/MAPK and PIK3CA/AKT to promote angiogenesis; increased VEGFA expression has been associated with ascites formation and poor prognosis (Prakash et al, 2010), and phase III trials have recently demonstrated significantly improved outcome in ovarian cancer patients receiving concomitant and maintenance bevacizumab, a humanised monoclonal antibody that inhibits VEGFA, in addition to standard first-line chemotherapy (Burger et al, 2011; Perren et al, 2011). 
$P D G F$ and associated receptors are also overexpressed in ovarian tumours, where increased expression promotes angiogenesis, in part by increasing VEGF production (Dong et al, 2004). Increased PDGFRA signalling is associated with more aggressive tumour phenotypes (Henriksen et al, 1993) and with reduced survival in ovarian cancer patients (Henriksen et al, 1993; Matei et al, 2006), and fibroblast growth factor (FGF) expression is also elevated in ovarian tumours (Fujimoto et al, 1997), in part because of an FGF1 gene amplification associated with increased angiogenesis and reduced patient survival (Birrer et al, 2007). Recent clinical trials of PDGF- and FGF-targeted drugs in ovarian cancer have demonstrated only modest survival benefits (Burger, 2011), possibly because of lack of target specificity resulting from degeneracy of growth factor signalling pathways or the development of drugs designed to simultaneously target multiple RTK pathways to maximise antiangiogenic effects (Ledermann and Raja, 2010; Burger, 2011; Pliarchopoulou and Pectasides, 2011). Development of patient selection or treatment response biomarkers is therefore challenging, particularly when RTK-targeted drugs are prescribed together with traditional cytotoxic chemotherapy.

Tumour-specific mutations are less common in ovarian cancer than in other solid tumours (Kalamanathan et al, 2011) and, therefore, unlike EGFR amplification to predict erlotinib sensitivity (Lynch et al, 2004) and the association of KRAS mutation status with cetuximab response (Garrett and Eng, 2011), they have limited applicability as response biomarkers. There is therefore an urgent clinical need to develop sensitive, quantitative assays to better subclassify ovarian tumours, more logically select individual patients most likely to respond to chemotherapy and to improve clinical trial design. We have therefore developed quantitative realtime PCR (qRT-PCR) methods to assess intertumour differences in the expression of candidate genes including growth factors, RTK drug targets, drug-metabolising enzymes and drug-resistance genes, and have correlated individuality in gene expression with ovarian tumour histology, chemotherapy response and patient survival.

\section{MATERIALS AND METHODS}

\section{Demographics of ovarian cancer patient cohorts}

Epithelial ovarian tumours of various histologies, obtained from chemotherapy-naive patients, were provided by the Edinburgh Experimental Cancer Medicine Centre ( $n=96$ fresh-frozen tumours, patients diagnosed between January 1990 and April 2006) and Tayside Tissue Bank $(n=91$ formalin-fixed, paraffin-embedded (FFPE) tumours, patients diagnosed between February 2005 and July 2009, Table 1, Supplementary Table S1). Tumour histology, FIGO stage and grade were assessed by experienced pathologists, and tumours subclassified as serous or nonserous (a combination of mucinous, endometrioid and clear-cell histologies). Age at diagnosis, the extent of surgical tumour debulking and chemotherapy response information was obtained from individual patient case notes. All patient recruitment was carried out following approval from the Lothian Research Ethics Committee (08/S1101/41) or the Tayside Tissue Bank Ethics Committee, a subcommittee of the Tayside Committee on Medical Research Ethics. Written informed consent was obtained from all study participants.

\section{Tissue processing and RNA extraction}

Fresh-frozen tissue Snap-frozen tumours $(\sim 20 \mathrm{mg})$ were suspended in lysis buffer (Qiagen, Crawley, UK) containing $1 \%$ $\beta$-mercaptoethanol and disrupted using a rotor-stator homogeniser (Kinematica, Lucerne, Switzerland). RNA extraction was performed using Qiagen RNeasy mini kits, according to the manufacturer's protocol for RNA extraction from animal tissues, extended to include on column DNAse digestions (RNAse free DNAse kit; Qiagen).
Table I Patient demographics

\begin{tabular}{|c|c|c|}
\hline & Fresh & FFPE \\
\hline \multicolumn{3}{|l|}{ Age at diagnosis (years) } \\
\hline Median & 63.5 & 63 \\
\hline Range & $37-90$ & $35-91$ \\
\hline \multicolumn{3}{|l|}{ PFS (days) } \\
\hline Median & 264.5 & 744 \\
\hline Range & $0-6356$ & $26-2202$ \\
\hline \multicolumn{3}{|l|}{ Histology } \\
\hline Serous & 59 & 65 \\
\hline Nonserous & 37 & 26 \\
\hline \multicolumn{3}{|l|}{ FIGO stage } \\
\hline I & 13 & 26 \\
\hline$\|$ & 9 & 13 \\
\hline III & 54 & 37 \\
\hline IV & 17 & 5 \\
\hline Unknown & 3 & 10 \\
\hline \multicolumn{3}{|l|}{ Grade of differentiation } \\
\hline Well & 3 & 11 \\
\hline Moderately & 13 & 10 \\
\hline Poorly & 75 & 55 \\
\hline Unknown & 5 & 15 \\
\hline \multicolumn{3}{|l|}{ Tumour debulking } \\
\hline Optimal debulking & 41 & 2 \\
\hline Partial debulking & 23 & 0 \\
\hline No debulking & 23 & 80 \\
\hline Unknown & 9 & 0 \\
\hline
\end{tabular}

Abbreviations: $\quad F F P E=$ formalin-fixed, $\quad$ paraffin-embedded; $\quad F \mid G O=$ International Federation of Gynaecology and Obstetrics; PFS = progression-free survival.

FFPE tissue Tumour-containing FFPE blocks were reviewed, selected and histology confirmed by an experienced pathologist (Professor CS Herrington). Three 20- $\mu \mathrm{m}$ sections were cut from each block and combined for RNA extraction, using an Ambion Recoverall Total Nucleic Acid Isolation Kit for FFPE tissues (Life Technologies, Paisley, UK), according to the manufacturer's instructions.

\section{Analysis of RNA yield and Integrity}

RNA yield and integrity was initially assessed from absorbance readings at 260 and $280 \mathrm{~nm}$ using a Nanodrop 1000 spectrophotometer (Thermo Fisher Scientific, Loughborough, UK). RNA integrity was further confirmed in RNA extracted from freshfrozen tumours using an Agilent Bioanalyzer 2100 and RNA 6000 Nano LabChip Kit (Agilent Technologies, Edinburgh, UK), according to the manufacturer's guidelines. The Agilent Bioanlyser calculates an RNA Integrity Number (RIN), ranging from 0 (completely degraded RNA) to 10 (fully intact RNA samples), and represents RNA integrity as a size-sorted electropherogram. Both RIN values (median 6.9, range 5.0-9.6) and electropherogram traces were used to select RNA samples of sufficient quality for gene expression analysis. RNA was initially extracted from 133 tumours - RNA from 13 tumours was of insufficient quality for further analysis, and full clinical information was available for 96 of the remaining 120 tumours. It was not possible to similarly assess RNA integrity in FFPE tumours $(n=91)$ using the Agilent Bioanalyzer because of the more limited size range of RNA fragments obtained from FFPE material.

\section{cDNA preparation}

RNA prepared from both fresh-frozen and FFPE tumours was reverse transcribed into cDNA (50 ng RNA per $20 \mu \mathrm{l}$ R reaction) 
using High Capacity RNA to cDNA kits (Applied Biosystems, Warrington, UK), according to the manufacturer's instructions.

\section{qRT-PCR analysis}

Fresh-frozen tissue cDNA prepared from RNA extracted from fresh-frozen tumours was analysed on Format 32 384-well Taqman Low Density Array (TLDA) cards (Applied Biosystems, Warrington, $\mathrm{UK}$ ), designed such that the expressions of 32 genes (31 target genes and $18 S$ ribosomal RNA as loading control, Supplementary Table S2) were simultaneously analysed in triplicate; four tumour samples were analysed on each card. Candidate genes for Taqman analysis were identified from PubMed searches using combinations of search terms including ovarian cancer, drug target, angiogenesis, signalling pathway, chemotherapy, response, resistance and platinum, inventoried Taqman probes identified (Supplementary Table S3) and TLDA cards manufactured by Applied Biosystems according to our specification.

PCR mixes were prepared by adding $10 \mu \mathrm{l}$ of each cDNA sample and $90 \mu \mathrm{l}$ of sterile water to $100 \mu \mathrm{l}$ of Taqman Universal PCR Master Mix (Applied Biosystems), and added to the appropriate TLDA sample ports. Following centrifugation $(2 \times 1 \mathrm{~min}$, 1200 r.p.m) to evenly distribute the reaction mixes, each card was sealed (TLDA card sealer; Applied Biosystems) and run on a Taqman 7900 real-time PCR system (Applied Biosystems) using standard pre-defined thermal cycling conditions $\left(50^{\circ} \mathrm{C}\right.$ for $2 \mathrm{~min}$, $94.5^{\circ} \mathrm{C}$ for $10 \mathrm{~min}$, and then 40 cycles of $97^{\circ} \mathrm{C}$ for $30 \mathrm{~s}$ and $59^{\circ} \mathrm{C}$ for $1 \mathrm{~min}$ ).

FFPE tissue Gene expression in FFPE tumours was analysed on 96-well plates, using individual Taqman assays optimised to work on FFPE material (Taqman amplicons limited to $100 \mathrm{bp}$ ). Analysis was restricted to genes (FGF1 and KIT) most significantly associated with tumour histology in our fresh-frozen tumour cohort, with the addition of WT-1 (Taqman probe ID Hs01103749_m1), FGFR1 (Taqman probe ID Hs00915142_m1) and FGFR2 (Taqman probe ID Hs01552926_m1). Analysis of KIT expression was performed using the same Taqman assay used in our TLDA card analysis (Supplementary Table S2); additional 'short-amplicon' Taqman assays were used for FGF1 (Taqman probe ID Hs00265254_m1) and 18 S ribosomal RNA (Taqman probe ID Hs03003631_g1).

For single probe analysis, $20 \mu \mathrm{l}$ individual reaction mixes (per well) contained $10 \mu \mathrm{l}$ Taqman Universal PCR Master Mix, $1 \mu \mathrm{l}$ gene-specific Taqman probe, $1 \mu \mathrm{l} \mathrm{cDNA}$ and $8 \mu \mathrm{l}$ sterile water. Each reaction was performed in triplicate and analysed on the Standard Real Time PCR programme on a 7900 Taqman real-time PCR system (Applied Biosystems), as described above.

Analysis of gene expression Analysis of both our single probe and TLDA card experiments was performed using SDS 2.3 software (Applied Biosystems). Optimal experimental baselines and thresholds were chosen for each gene, and gene expression in individual tumour samples quantitated by cycle threshold $(\mathrm{Ct})$ values. Relative expression values were determined by comparing the expression of each target gene with the invariant 'loading control' 18 S ribosomal RNA, as previously described (Smith et al, 2003a,b). All samples were analysed in triplicate and gene expression calculated relative to $18 \mathrm{~S}$ ribosomal RNA \pm compound error ( $($ s.d. target gene $\left.)^{2}+(\text { s.d. } 18 \mathrm{~S} \text { ribosomal RNA })^{2}\right)^{1 / 2}$, where s.d. is the standard deviation of the mean of triplicate replicates.

\section{FGF1 gene copy number assay}

To assess FGF1 copy number, DNA was extracted from our freshfrozen tumour cohort $(n=88)$ using an Ambion Recoverall Total nucleic acid isolation kit from FFPE (Life Technologies) according to the manufacturer's instructions, and DNA concentration determined using a Nanodrop 1000 spectrophotometer (Thermo Scientific). Copy number was assessed using a quantitative Taqman gene copy number assay (Taqman assay ID Hs05994446_cn), where FGF1 copy number was compared with the endogenous control gene RNAse $P$ (copy number 2) by the comparative $\mathrm{Ct}$ method, and relative quantitation values obtained using CopyCaller Software (Applied Biosystems). As additional controls, FGF1 and RNAse $P$ copy numbers were assessed in peripheral blood samples $(n=4)$, and copy numbers of 2 confirmed.

\section{Correlation of gene expression with tumour histology, chemotherapy response and patient survival}

All statistical tests were performed using the PASW statistics package version 18.0 (IBM, New York, USA). Kruskal-Wallis oneway analysis of variance tests were performed to test for differences in gene expression between different tumour histologies, in patients with platinum-sensitive and -resistant disease and in platinum-sensitive and -resistant cell lines. Spearman's rank correlation analysis was used to investigate relationships between the expression of individual genes, and the significance of the Spearman's co-efficient $r^{2}$ assessed using Fisher's transformation tests. Log-rank analysis and Kaplan-Meier plots were performed to correlate patient survival with gene expression, and the influence of potential confounding factors including age at diagnosis, tumour histology, tumour stage and grade and extent of surgical tumour debulking evaluated using Cox regression analysis.

\section{Immunohistochemical analysis of WT-1 expression}

Sections $(4 \mu \mathrm{M})$ from FFPE tumours were cut onto Superfrost plus slides (VWR International Ltd, Lutterworth, UK) and dried for $1 \mathrm{~h}$ at $60{ }^{\circ} \mathrm{C}$ before de-paraffinisation in Histoclear (Fisher Scientific) and rehydration through a graded alcohol series. Sections were microwaved for 10-min before immunostaining on a DAKO autostainer (Dako, Ely, UK) using Vectastain ABC kits (Vector Labs, Peterborough, UK) according to the manufacturer's protocol. Sections were blocked in horse serum containing $10 \%(\mathrm{v} / \mathrm{v})$ avidin solution (Vector Labs) for $20 \mathrm{~min}$ followed by a 1-h incubation with $1 / 50$ dilution of $W T-1$ primary antibody (clone $6 \mathrm{~F}-\mathrm{H} 2$; DAKO, Ely, UK) including $10 \%(\mathrm{v} / \mathrm{v})$ biotin solution (Vector Labs). Sections were then incubated with biotinylated anti-mouse antibody for $30 \mathrm{~min}$ followed by Vectastain Elite $\mathrm{ABC}$ reagent for $30 \mathrm{~min}$. Liquid diaminobenzidine (DAKO) was used as a chromogenic agent for $5 \mathrm{~min}$ and sections counterstained with Mayer's haematoxylin. Sections known to stain positively were included in each batch, and negative controls prepared by replacing the primary antibody with TBS buffer.

\section{Analysis of FGF1 expression in drug-sensitive and drug-resistant ovarian cell lines}

Paired drug-sensitive and drug-resistant cell lines were obtained from Dr Simon Langdon, University of Edinburgh (PEO1/PEO4 and PEA1/PEA2; Langdon et al, 1988) or ATCC, LGC Standards, Teddington, UK (A2780/A2780DPP; Lu et al, 1988). The PEO and PEA series cell lines were derived from ascites fluid obtained from ovarian cancer patients before and after the development of drug resistance, whereas the drug-resistant A2780DPP cell line was derived in vitro, following continuous selection in increasing concentrations of cisplatin. PEO1 and PEO4 cells were maintained in RPMI-1640 supplemented with 15\% FBS, $0.1 \%$ glucose, $25 \mathrm{mmoll}^{-1}$ Hepes and $10 \mathrm{mgl}^{-1}$ insulin, PEA1, PEA2 and A2780 cells in RPMI-1640 supplemented with 10\% FBS, and A2780DPP cells in RPMI-1640 supplemented with 15\% FBS and $1 \mu \mathrm{M}$ cisplatin in $37^{\circ} \mathrm{C}$ incubators, supplemented with $5 \% \mathrm{CO}_{2}$. Cells were grown to $80 \%$ confluency in $75 \mathrm{~cm}^{2}$ flasks, harvested by 
trypsinisation, counted using a haemocytometer and $1 \times 10^{7}$ cells used for RNA extraction using a Qiagen RNeasy mini kit (Qiagen), following the manufacturer's protocol for mammalian cells, with on column DNAse digestion as described previously. The cDNA was prepared as described previously and the expression of FGF1 and $18 S$ ribosomal RNA assessed by qRT-PCR analysis.

\section{Creation and characterisation of stable FGF1 knockdown ovarian cell lines}

FGF1 expression was stably knocked down in A780DPP cells by RNA interference using Mission shRNA plasmids (Sigma-Aldrich, Gillingham, UK). Five unique FGF1-specific shRNA plasmids (clones A-TRC0000072524, B-TRC0000222594, C-TRC0000222593, D-TRC0000072527 and E-TRC0000072525) and a negative control plasmid were purchased as glycerol stocks and plasmid DNA extracted using plasmid DNA maxi prep kits (Qiagen), according to the manufacturer's instructions. A2780DPP cells $\left(2.5 \times 10^{5}\right.$ cells per well in 6-well plates) were transfected with each plasmid using lipofectamine (Invitrogen, Paisley, UK), and shRNA-containing cells selected with puromycin, according to the manufacturer's guidelines. Individual cell colonies were picked using cloning cylinders, expanded to $75 \mathrm{~cm}^{2}$ tissue culture flasks and screened for FGF1 expression by qRT-PCR analysis, as previously described. FGF1 knockdown was confirmed using a quantitative Human FGF acidic Quantikine Immunoassy (R\&D Systems, Inc., Abingdon, UK) to compare FGF levels in cell supernatants according to the manufacturer's guidelines.

\section{Ovarian cell line chemosensitivity assays}

MTT assays (Mosmann, 1983) were used to compare the chemosensitivity of A2780DPP and FGF1 knockdown cells to cisplatin and carboplatin. Each cell line was plated in a 96-well plate (5000 cells per well) and treated in triplicate with serial dilutions of each drug, with drug concentrations selected to mimic typical peak plasma levels in ovarian cancer patients (range $0-200 \%$ peak plasma; cisplatin $0.8-25 \mu \mathrm{M}$, carboplatin $2.7-85 \mu \mathrm{M}$ ). Cells were treated for $72 \mathrm{~h}$, media removed and $100 \mu \mathrm{l}$ of a $0.5 \mathrm{mg} \mathrm{ml}^{-1} \quad$ MTT solution (3-(4,5-dimethylthiazol-2-yl)-2,5diphenyltetrazolium bromide in phenol red free DMEM) added and cells incubated at $37^{\circ} \mathrm{C}$ for $3 \mathrm{~h}$. The resulting formazan crystals were solubilised in DMSO, quantitated spectrophotometrically at $570 \mathrm{~nm}$ and the percentage of live cells remaining following each drug treatment calculated (assigning a value of $100 \%$ to vehicletreated cells). The $\mathrm{IC}_{50}$ values were calculated from log dose response curves.

\section{RESULTS}

\section{qRT-PCR analysis of individuality in gene expression}

In initial experiments, we assessed intertumour variation in the expression of 31 candidate genes including growth factors (FGF1, FGF2, KITLG, VEGF), RTKs (CSF1R, KIT, EGFR, IGFR, FLT3, $E R B B, \quad P D G F R, V E G F R)$, key nodes in signalling pathways (PIK3CA, PTEN, AKT2, FRAP1, SRC, JUN) or mediators of apoptosis ( $p 53, M D M 2, X I A P)$ and drug resistance $(A B C B 1$, $A B C C 2$, GSTP1) (Supplementary Tables S2 and S3). qRT-PCR analysis (format 32 TLDA cards) was used to compare gene expression, relative to the loading control $18 S$ ribosomal RNA, in unselected fresh-frozen ovarian tumours $(n=96)$, as described in Materials and Methods. Marked intertumour expression differences were observed (see, e.g., Figure 1A-D), with each tumour having a unique expression profile. GSTP1 was the most abundantly expressed gene in the majority of tumours, although more than 70 -fold variation in GSTP1 expression was observed (Figure 1E). Similar intertumour variations in gene expression were observed for all genes, and absolute gene expression varied widely (Figure $1 \mathrm{~F}$ and Supplementary Figure S1). Pair-wise comparisons of gene expression revealed significant correlations between the RTKs PDGFRA and PDGFRB $\left(r^{2}=0.781, P<0.001\right)$, VEGFR1 (FLT1) and VEGFR2 (KDR) $\left(r^{2}=0.698, P<0.001\right)$ and VEGFR2 and PRGFRB $\left(r^{2}=0.716, P<0.001\right)$.

\section{Correlation of gene expression with clinical parameters}

Tumour histology To assess whether intertumour variation in gene expression correlated with histology, we compared gene expression in serous $(n=59)$ and nonserous $(n=37)$ tumours. To validate tumour histology, we first compared WT-1 expression (Figure 2A) and confirmed previous reports of increased expression in serous tumours $(P=0.001, P=0.031$ adjusted for multiple testing). We identified an additional 6 genes $\left(F G F 1\left(P=1.7 \times 10^{-5}\right.\right.$, adjusted $\left.P=5.27 \times 10^{-4}\right), \quad E R B B 4 \quad(P=0.004)$, JUN $(P=0.006)$, $\operatorname{VEGFB}(P=0.014), \operatorname{CSF} 1 R(P=0.03)$ and $\operatorname{KDR}(P=0.079))$ more highly expressed in serous tumours and 3 genes $(K I T(P=0.005)$, IGF2R $(P=0.04)$ and $A B C C 2(P=0.039))$ more highly expressed in nonserous tumours (Figure 2 and Supplementary Figure S2).

\section{Patient survival}

To assess whether gene expression influenced survival, progression-free survival intervals were calculated for all patients (days between the end of the first course of chemotherapy and disease progression or death) and compared with quartiles of gene expression. FGF1 (adjusted log-rank test $P=0.006$ ) and $P D G F R B$ (adjusted log-rank test $P=0.037$ ) expression were significantly inversely associated with survival in multivariate analysis (Figure 3), where age, tumour histology, stage, grade and the extent of tumour debulking were considered as possible confounders. Of these variables, only tumour stage was independently associated with progression-free survival $(P=0.008)$. $F G F 1$, but not $P D G F R B$, expression was also significantly inversely associated with overall patient survival (adjusted log-rank test $P=0.008$, Supplementary Figure S3).

As FGF1 gene amplification was previously associated with survival (Birrer et al, 2007), we used quantitative Taqman gene copy number assays to investigate whether individuality in FGF1 mRNA expression correlated with copy number variation. As described in Materials and Methods, FGF1 copy number was compared with endogenous control gene RNAse P (copy number 2), and additionally with DNA from a reference blood sample with a diploid genome. No FGF1 copy number changes were identified (data not shown).

Correlation of gene expression with chemotherapy response Response to first-line platinum-based chemotherapy, where patients are defined as 'sensitive' if they relapse $>6$ months after completion of treatment and 'resistant' if relapse occurs $<6$ months, is a significant determinant of subsequent chemosensitivity and patient survival (Blackledge et al, 1989). To investigate whether gene expression influenced chemosensitivity, we restricted our analysis to a subgroup of 74 patients treated with either single-agent cisplatin or carboplatin, or platinum and paclitaxel drug combinations (additional patients had not received chemotherapy or were not treated with platinum drugs; (Supplementary Table S1A); therefore, our gene expression comparisons in drug-sensitive and drug-resistant patients had limited power. Interestingly, however, MDM2 $(P=0.032)$ and ERBB2 $(P=0.064)$ expression was higher in platinum-sensitive patients, highlighting associations that will be prioritised for future analysis in larger patient cohorts. 

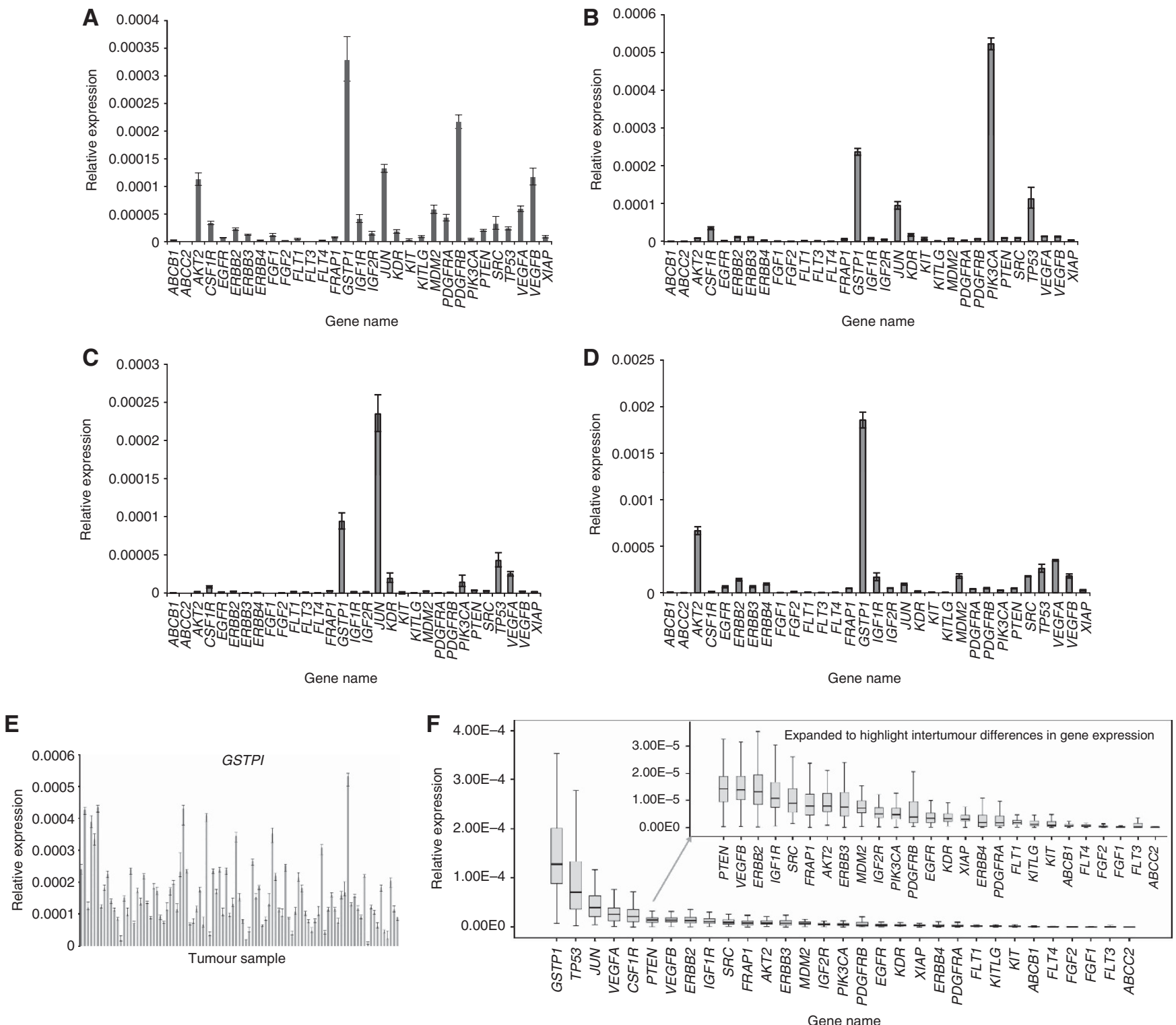

Figure I TLDA cards were used to compare the expression of 3 I candidate genes, relative to the expression of the loading control I 8S ribosomal RNA, as described in Materials and Methods. Gene expression was assessed in fresh-frozen ovarian tumours $(n=96)$, and is represented as mean \pm s.d. of triplicate replicates. (A-D) Gene expression in 4 representative ovarian tumours; (E) intertumour variation in GSTPI expression, where each bar represents one tumour; (F) summary of variation in relative gene expression (box plot median) and the extent of intertumour variability in the expression of each gene (box plot whiskers).

\section{Validation of clinical associations}

As these associations may help rationalise individuality in disease progression and/or facilitate patient selection for targeted chemotherapy, we extended our analysis to an additional cohort of FFPE tumours to confirm our findings in archival material. As described in Materials and Methods, we limited our PCR amplicons to $100 \mathrm{bp}$, and performed pilot experiments to confirm similar expression in matched fresh-frozen and FFPE tumours (data not shown). We then investigated intertumour expression differences in WT-1, FGF1 and KIT (genes that had previously shown the most significant associations with tumour histology) in FFPE tumours $(n=91)$. Again, marked intertumour differences in gene expression and significant associations with tumour histology were observed (Figure 4), where $W T-1(P<0.001$, Figure $4 \mathrm{~A})$ and $F G F 1$ ( $P=0.003$, Figure 4 C) were more highly expressed in serous tumours and $\operatorname{KIT}(P=0.013$, Figure $4 \mathrm{E})$ in nonserous tumours. Increased $W T-1$ expression in serous tumours was confirmed by immunohistochemistry using an antibody validated for routine tumour histopathology (Figure 4B).

FGF1 activates signal transduction by binding to the RTKs FGFR1 and FGFR2 (Turner and Grose, 2010). We therefore additionally assessed individuality in FGF receptor expression in both tumour cohorts, and again found marked intertumour expression differences. Furthermore, there was a significant correlation between FGF1 and FGFR2 $(P=0.026)$, but not FGFR1 $(P=0.214)$ expression and, similar to $F G F 1, F G F R 2$ was more highly expressed in serous tumours $(P=0.003$, Figure 4D). FGFR2 expression was also significantly inversely correlated with progression-free survival in our fresh-frozen tumour cohort (adjusted log-rank test $P=0.04$ ), which was again independently influenced by tumour stage $(P=0.05)$. 

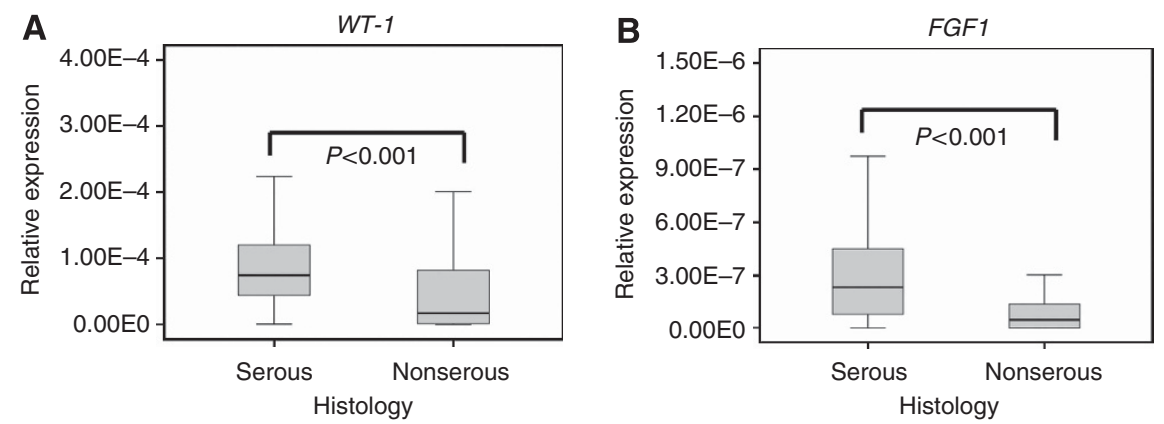

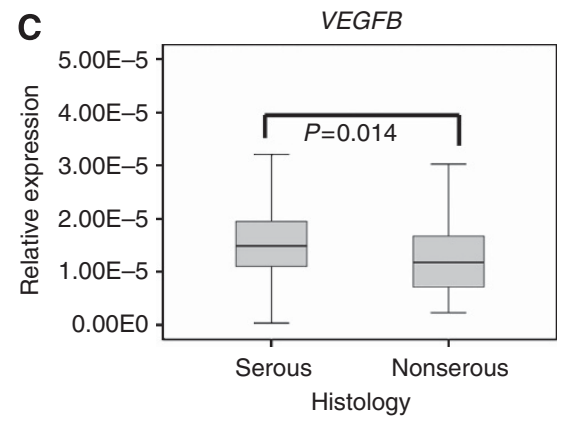

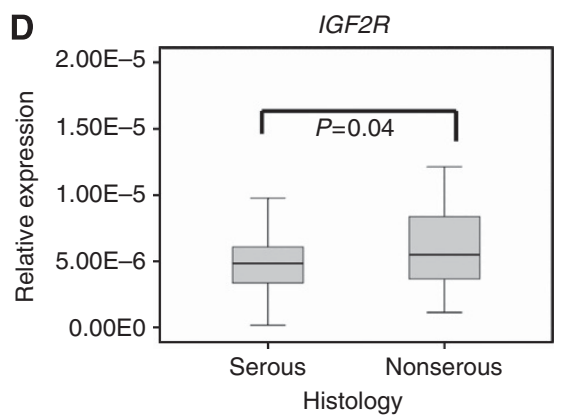

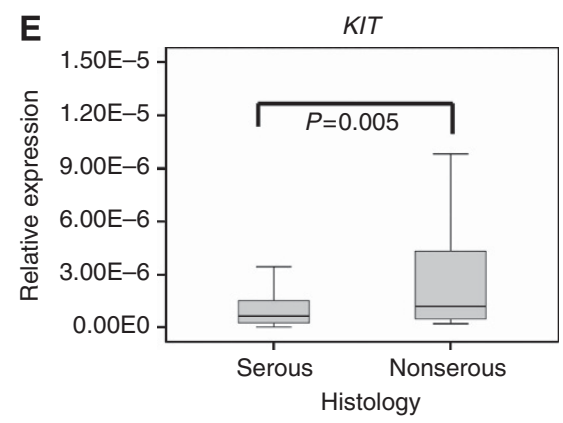

Figure 2 Association of gene expression with tumour histology. TLDA cards were used to compare gene expression in fresh-frozen ovarian tumours $(n=96)$ as described in Materials and Methods, and tumours subclassified according to histology - serous tumours $(n=59)$ and nonserous tumours $(n=37$, combined mucinous, endometrioid and clear-cell histologies). WT-I (A), FGFI (B) and VEGFB (C) were significantly overexpressed in serous tumours; IGF2R (D) and KIT (E) were significantly overexpressed in nonserous tumours. Significant differences in ABCC2, CSFIR, ERBB4, JUN and KDR gene expression were also observed (Supplementary Data Figure S2).

A

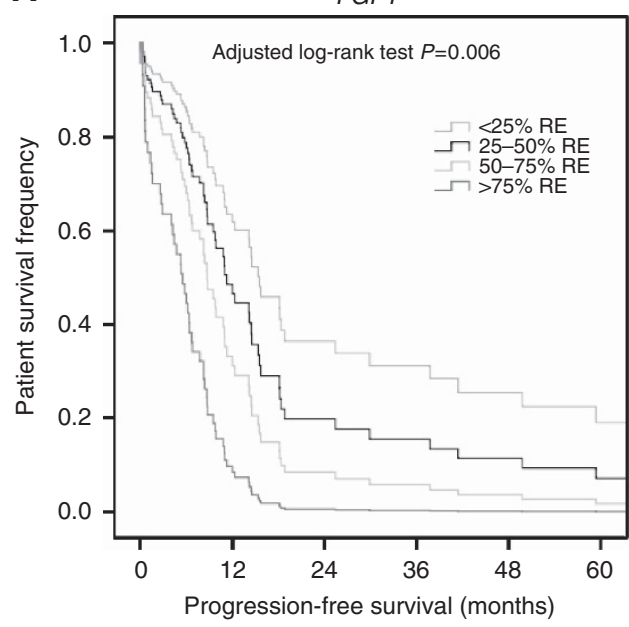

\begin{tabular}{cccc}
$\begin{array}{c}\text { Quartile range of } \\
\text { FGF1 expression (\%) }\end{array}$ & $\begin{array}{c}\text { Median survival } \\
\text { (days) }\end{array}$ & Hazard ratio & 95\% Cl \\
\hline$<25$ & 465.5 & 1 & \\
$25-50$ & 334 & 1.591 & $0.612-4.133$ \\
$50-75$ & 205.5 & 2.434 & $0.991-5.975$ \\
$>75$ & 188 & 5.123 & $2.015-13.028$ \\
\hline
\end{tabular}

B

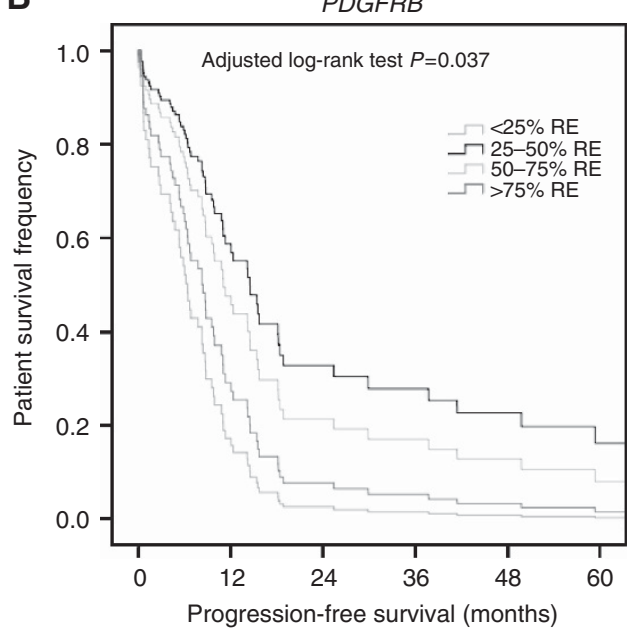

\begin{tabular}{cccc}
$\begin{array}{c}\text { Quartile range of } \\
\text { PDGFRB expression (\%) }\end{array}$ & $\begin{array}{c}\text { Median survival } \\
\text { (days) }\end{array}$ & Hazard radio & $95 \% \mathrm{Cl}$ \\
\hline$<25$ & 375 & 1 & \\
$25-50$ & 403 & 0.722 & $0.266-1.96$ \\
$50-75$ & 277 & 2.373 & $0.92-6.124$ \\
$>75$ & 198 & 1.664 & $0.745-3.721$ \\
\hline
\end{tabular}

Figure 3 Correlation of (A) FGFI and (B) PDGFRB expression with patient survival. Log-rank analysis was used to compare quartiles (0-25\%, 25-50\%, $50-75 \%$ and $75-100 \%$ of maximum expression) of FGFI and PDGFRB expression with progression-free survival, assessed from the end of the first course of chemotherapy, as described in Materials and Methods. Survival was adjusted for patient histology, the extent of debulking surgery and tumour histology, stage and grade in multivariate analysis. Correlation of FGFI expression with overall survival is illustrated in Supplementary Data Figure S3.

\section{FGF1 knockdown influences platinum chemosensitivity}

To investigate whether FGF1 also influenced platinum resistance, we used qRT-PCR analysis to compare FGF1 expression in paired platinum-sensitive and -resistant ovarian tumour cell lines, and found that FGF1 was consistently more highly expressed in platinum-resistant cells (Figure 5A). As only the A2780/A2780DPP cell line pair modelled single-agent platinum resistance (Lu et al, 1988), 
A

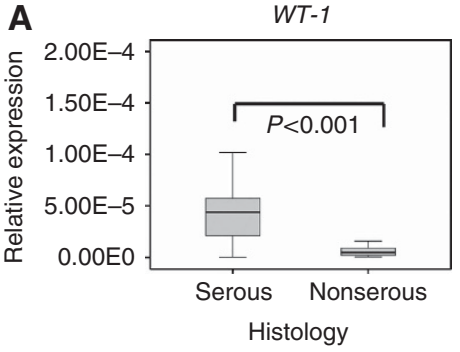

B

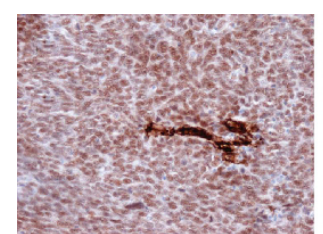

$W T-1$ serous $(\times 20)$

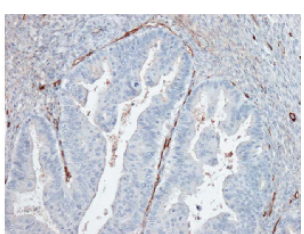

$W T-1$ mucinous $(\times 20)$
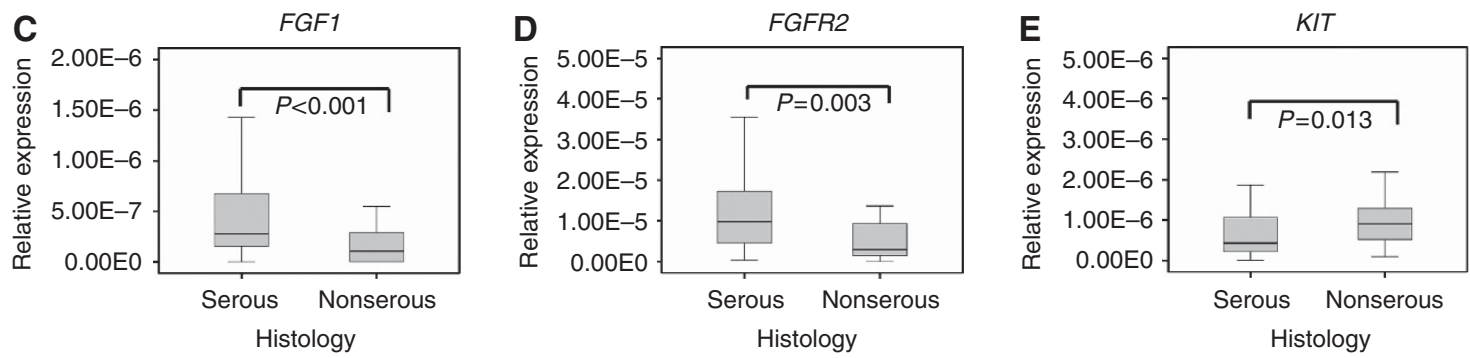

Figure 4 Validation of ovarian tumour histology associations. Single-gene Taqman probes were used to compare gene expression in FFPE ovarian tumours $(n=91)$ as described in Materials and Methods, and tumours subclassified according to histology - serous tumours ( $n=58)$ and nonserous tumours ( $n=33$, combined mucinous, endometrioid and clear-cell histologies). WT-I (A), FGFI (C) and FGFR2 (D) were significantly more highly expressed in serous tumours; increased WT-I expression in serous tumours was confirmed by immunohistochemical analysis as described in Materials and Methods (B). In contrast, KIT (E) was significantly overexpressed in nonserous tumours.

A

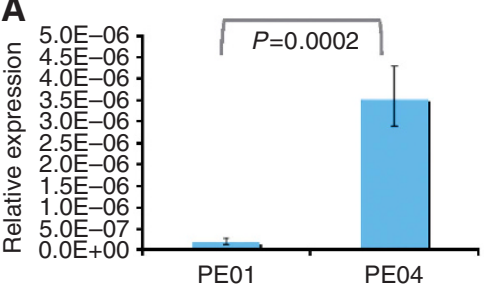

B
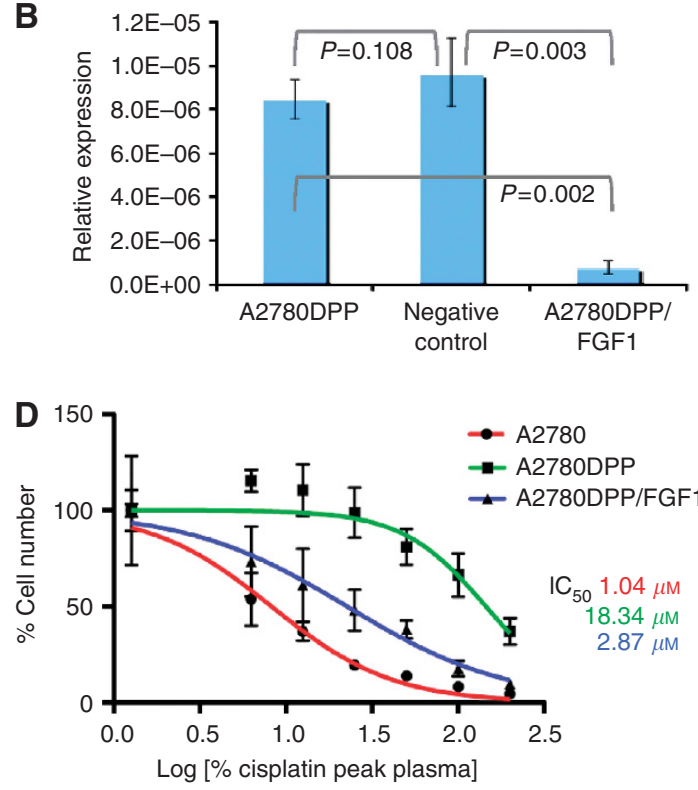
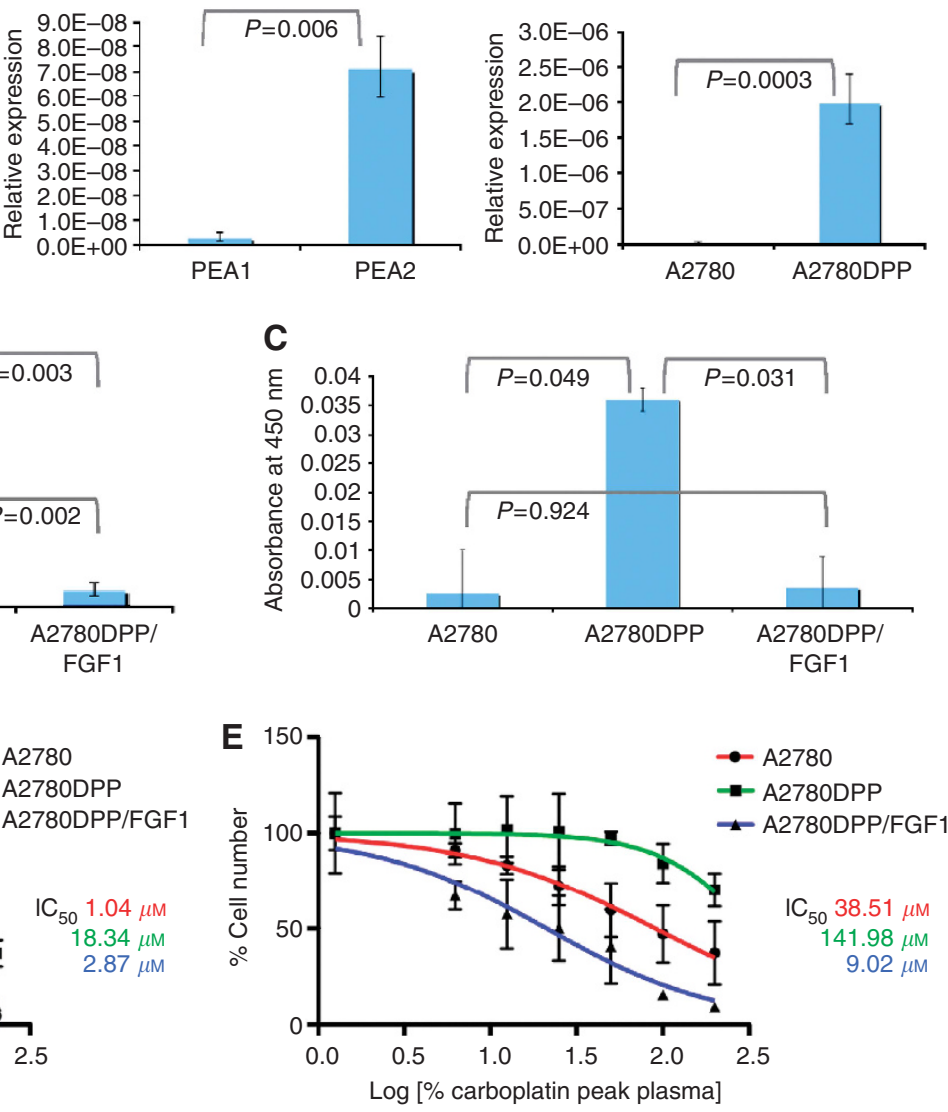

Figure $5 \mathrm{FGFI}$ expression influences platinum chemosensitivity. qRT-PCR analysis was used to compare FGF/ expression in paired platinum-sensitive and -resistant ovarian cancer cell lines, as described in Materials and Methods (A). FGFI expression was stably knocked down in A2780DPP cells and reduction in gene expression confirmed by qRT-PCR analysis (B) and quantitative immunoassay (C). MTT assays were used to compare chemosensitivity to (D) cisplatin and (E) carboplatin in drug-sensitive A2780 cells, drug-resistant A2780DPP cells and A2780DPP/FGFI knockdown cells, as described in Materials and Methods. 
we stably knocked down FGF1 expression in A2780DPP cells. As described in Materials and Methods, we isolated three independent clones from each of five FGF1-specific shRNA plasmids and compared FGF1 expression in all 15 cell lines by qRT-PCR analysis (data not shown). The cell line derived from clone A2 (A2780DPP/FGF1) resulted in the most significant FGF1 knockdown, as assessed by both qRT-PCR analysis (Figure 5B) and immunoassay (Figure 5C), and was therefore used in subsequent experiments.

MTT assays were used to compare platinum chemosensitivity in A2780, A2780DPP and A2780DPP/FGF1 cells and significant differences in sensitivity to both cisplatin (Figure 5D) and carboplatin (Figure 5E) identified; A2780DPP cells were 17.6-fold resistant to cisplatin and 3.7-fold cross-resistant to carboplatin. FGF1 knockdown significantly re-sensitised A2780DPP cells to cisplatin with a corresponding $84.4 \%$ decrease in $\mathrm{IC}_{50}$, and resulted in a complete reversal of carboplatin resistance, where A2780DPP/ FGF1 cells had a significantly lower carboplatin $\mathrm{IC}_{50}(9.02 \mu \mathrm{M})$ than both the platinum-sensitive $\mathrm{A} 2780(38.51 \mu \mathrm{M})$ and parental platinum-resistant A2780DPP $(141.98 \mu \mathrm{M})$ cell lines.

\section{DISCUSSION}

Our data describe marked intertumour differences in the expression of several clinically relevant genes, and highlight novel associations with tumour histology, chemotherapy response and survival. Each tumour had a unique gene expression profile, consistent with individuality in both disease progression and treatment response, highlighting the potential for future development of more personalised treatment approaches.

Our gene expression/tumour histology associations identify a clinical variable that is routinely assessed but not routinely used as a treatment selection biomarker. We showed significant differences in the expression of several genes including FGF1 and KIT, which are targets for currently licenced chemotherapy drugs or drugs in clinical trial, highlighting the potential utility of tumour histology as a validated biomarker and important co-variate in clinical trial design. We emphasise the importance of extending our analysis to additional genes, and to clear-cell, mucinous and endometrioid tumours - analysis of archival FFPE material may facilitate tumour selection for future more complex array-based experiments and multicentre collaborative patient recruitment. Recently published whole genome microarray-based analyses from the Australian Ovarian Cancer Study (AOCS; Tothill et al, 2008) illustrate both the power of collaborative analysis and the necessity for comprehensive clinical annotation, and have resulted in the identification of novel molecular subtypes of both serous and endometrioid ovarian cancers associated with poor prognosis (Tothill et al, 2008; Konstantinopoulos et al, 2011).

Our data identify significant inverse relationships linking FGF1 expression with survival, highlighting functionally important pathways that may influence tumour progression, and prioritising targets for drug development. It was not possible to assess the influence of individuality in gene expression on patient survival in our FFPE tumour cohort, as the majority of patients had not reached a 5-year census point post diagnosis. It is tempting to speculate, however, that the striking similarities in gene expression and our confirmation of highly significant histology associations in fresh-frozen and FFPE tumours will, in time, also be replicated in common survival associations. To support this hypothesis, we investigated associations between FGF1 and PDGFRB expression and both progression-free and overall survival in a published data set (Affymetrix U133 Plus 2 arrays, Affymetrix, Santa Clara, CA, USA) of 285 high-grade, advanced-stage serous ovarian, fallopian tube and peritoneal tumours from the AOCS (Tothill et al, 2008). In support of our findings, FGF1 expression was significantly inversely associated with progression-free (time to relapse) and overall (time to death) survival in each of three FGF1 probe sets (adjusted $P<0.001$ and $P=0.030$, respectively) and, in multivariate analysis, was influenced by tumour stage, but not by tumour grade, histology or age at diagnosis. PDGFRB expression was also significantly associated with survival in unadjusted data from the AOCS data set $(P<0.001)$, but the association was not significant $(P=0.098)$ in multivariate analysis, where survival was significantly influenced by tumour stage $(P=0.02)$.

Our analysis has not only quantitatively described intertumour expression differences, but also delineated the relative importance of highly homologous RTK pathway members. In particular, FGF1 (but not FGF2) and FGFR2 (but not FGFR1) are differentially expressed in serous and nonserous ovarian tumours. As FGFR2 expression also significantly influences survival, we hypothesise that FGF1-dependent signalling in serous ovarian tumours may be initiated by FGFR2 binding - we are currently testing this hypothesis, and further evaluating the influence of FGF signalling on cell proliferation and invasion. Consistent with our findings, Cole et al (2010) have shown that FGFR2 knockdown reduced cell proliferation and increased platinum chemosensitivity in ovarian tumour cell lines and xenografts. As clinical practice increasingly moves to the use of neoadjuvant chemotherapy, we highlight the need to compare FGF1 and FGFR2 expression in chemotherapy-naive, platinum-sensitive and platinum-resistant ovarian cancer patients. Importantly, our findings that (1) FGF1 expression is increased in platinum-resistant ovarian tumour cell lines, and (2) FGF1 knockdown re-sensitises ovarian cancer cells to platinum drugs are entirely consistent with an inverse association between FGF1 expression and survival in ovarian cancer patients, and further supports the development of FGF1-specific inhibitors. FGF1, together with VEGF and PDGF, is a target for several pan-RTK drugs including cediranib (Astra Zeneca, Alderley Park, UK), pazopanib (GlaxoSmithKline, Brentford, UK) and BIBF 1120 (Boehringer Ingelheim, Bracknell, UK), although no FGF1-specific agents are currently licenced. We therefore highlight the need to further evaluate FGF1 and FGFR2 as biomarkers of platinum sensitivity in larger independent patient cohorts. Should our associations with platinum chemosensitivity be confirmed, it will be of obvious interest to investigate whether quantitative immunoassay-based assessment of serum FGF1 levels may have clinical utility as a drug resistance biomarker.

Although our power to detect associations between gene expression and chemotherapy response was limited, our data suggest that $M D M 2$ and $E R B B 2$ expression may also influence platinum sensitivity in chemotherapy-naive patients. In support of these findings, $M D M 2$ overexpression has been shown to sensitise A2780 cells to cisplatin ( $\mathrm{Mi}$ and Ni, 2003), whereas reduced MDM2 expression (inheritance of SNP309) was associated with increased survival in ovarian cancer patients (Bartel et al, 2008). In contrast to our data, however, clinical trials of the ERBB2 monoclonal antibody inhibitor pertuzumab describe enhancement of carboplatin efficacy in ovarian tumours, and increased platinum sensitivity in uterine (Cross et al, 2010) tumours overexpressing ERBB2.

We have additionally described marked intertumour differences in the expression of VEGFA, EGFR, IGFR, PIK3CA and SRC, targets for newly licenced chemotherapy drugs (Banerjee and Kaye, 2011). It will therefore be of interest in future studies to investigate whether clinical response is influenced by individuality in gene expression and/or by tumour histology. Individuality in GSTP1 expression has also previously been associated with platinum sensitivity, and with the drug-resistance phenotype in ovarian cancer cell lines and patients (Townsend and Tew, 2003; PeklakScott et al, 2008); we are currently investigating whether, similar to FGF1, GSTP1 directly influences platinum chemosensitivity. We also found marked inter-tumour variability in expression of the 'drug resistance' genes $A B C B 1$ (MDR1) and ABCC2 (MRP2), 
previously associated with platinum ( $A B C B 1$ and $A B C C 2)$, doxorubicin and vincristine (primarily $A B C B 1$ )-induced drug resistance in ovarian cancer patients (Bourhis et al, 1989; Ohishi et al, 2002). Although the development of drug resistance occurs as an adaptive response to chemotherapy, our analysis of tumours from chemotherapy-naive patients suggests that individual tumours may, before treatment, have inherent differences in drug sensitivity.

In summary, therefore, we describe marked individuality in the expression of clinically relevant genes in ovarian tumours, and highlight novel associations with tumour histology and survival, consistent with individuality in disease progression and treatment response. We show for the first time that FGF1 is differentially expressed in aggressive high-grade serous ovarian tumours, and that FGF1 expression is a significant determinant of survival and response to platinum-based chemotherapy.

\section{REFERENCES}

Al-Hussaini M, Stockman A, Foster H, McCluggage WG (2004) WT-1 assists in distinguishing ovarian from uterine serous carcinoma and in distinguishing between serous and endometrioid ovarian carcinoma. Histopathology 44(2): 109-115

Banerjee S, Kaye S (2011) The role of targeted therapy in ovarian cancer. Eur J Cancer 47(Suppl 3): S116-S130

Bartel F, Jung J, Bohnke A, Gradhand E, Zeng K, Thomssen C, Hauptmann S (2008) Both germ line and somatic genetics of the p53 pathway affect ovarian cancer incidence and survival. Clin Cancer Res 14(1): 89-96

Birrer MJ, Johnson ME, Hao K, Wong KK, Park DC, Bell A, Welch WR, Berkowitz RS, Mok SC (2007) Whole genome oligonucleotide-based array comparative genomic hybridization analysis identified fibroblast growth factor 1 as a prognostic marker for advanced-stage serous ovarian adenocarcinomas. J Clin Oncol 25(16): 2281-2287

Blackledge G, Lawton F, Redman C, Kelly K (1989) Response of patients in phase II studies of chemotherapy in ovarian cancer: implications for patient treatment and the design of phase II trials. Br J Cancer 59(4): 650-653

Bourhis J, Goldstein LJ, Riou G, Pastan I, Gottesman MM, Benard J (1989) Expression of a human multidrug resistance gene in ovarian carcinomas. Cancer Res 49(18): 5062-5065

Brown MR, Blanchette JO, Kohn EC (2000) Angiogenesis in ovarian cancer. Baillieres Best Pract Res Clin Obstet Gynaecol 14(6): 901-918

Burger RA (2011) Overview of anti-angiogenic agents in development for ovarian cancer. Gynecol Oncol 121(1): 230-238

Burger RA, Brady MF, Bookman MA, Fleming GF, Monk BJ, Huang H, Mannel RS, Homesley HD, Fowler J, Greer BE, Boente M, Birrer MJ, Liang SX (2011) Incorporation of bevacizumab in the primary treatment of ovarian cancer. $N$ Engl J Med 365(26): 2473-2483

Clark TG, Stewart ME, Altman DG, Gabra H, Smyth JF (2001) A prognostic model for ovarian cancer. Br J Cancer 85(7): 944-952

Cole C, Lau S, Backen A, Clamp A, Rushton G, Dive C, Hodgkinson C, McVey R, Kitchener H, Jayson GC (2010) Inhibition of FGFR2 and FGFR1 increases cisplatin sensitivity in ovarian cancer. Cancer Biol Ther 10(5): 495-504

Cross SN, Cocco E, Bellone S, Anagnostou VK, Brower SL, Richter CE, ER Siegel, Schwartz PE, Rutherford TJ, Santin AD (2010) Differential sensitivity to platinum-based chemotherapy in primary uterine serous papillary carcinoma cell lines with high vs low HER-2/neu expression in vitro. Am J Obstet Gynecol 203(2): 162 e1-162 e8

Dong J, Grunstein J, Tejada M, Peale F, Frantz G, Liang WC, Bai W, Yu L, Kowalski J, Liang X, Fuh G, Gerber HP, Ferrara N (2004) VEGF-null cells require PDGFR alpha signaling-mediated stromal fibroblast recruitment for tumorigenesis. EMBO J 23(14): 2800-2810

Fujimoto J, Ichigo S, Hori M, Hirose R, Sakaguchi H, Tamaya T (1997) Expression of basic fibroblast growth factor and its mRNA in advanced ovarian cancers. Eur J Gynaecol Oncol 18(5): 349-352

Garrett CR, Eng C (2011) Cetuximab in the treatment of patients with colorectal cancer. Expert Opin Biol Ther 11(7): 937-949

Henriksen R, Funa K, Wilander E, Backstrom T, Ridderheim M, Oberg K (1993) Expression and prognostic significance of platelet-derived growth factor and its receptors in epithelial ovarian neoplasms. Cancer Res 53(19): 4550-4554

\section{ACKNOWLEDGEMENTS}

We thank the research nurses and technical staff in Tayside Tissue Bank for help with patient recruitment, sample provision and compilation of clinical response databases, Dr Susan Bray for performing WT-1 immunohistochemistry and Dr Neil Kernohan for helpful discussions. We additionally acknowledge Professor John Smyth who created the Edinburgh Ovarian Cancer database and Tyyzia Rye for ongoing database maintenance. We gratefully acknowledge the award of a Strategic Research Development Grant from the Scottish Funding Council and additional funding from Cancer Research UK (C4639/A10822). C Gourley is funded by a Scottish Senior Clinical Fellowship.

Supplementary Information accompanies the paper on British Journal of Cancer website (http://www.nature.com/bjc)

Kalamanathan S, Bates V, Lord R, Green JA (2011) The mutational profile of sporadic epithelial ovarian carcinoma. Anticancer Res 31(8): 2661-2668

Kobel M, Kalloger SE, Boyd N, McKinney S, Mehl E, Palmer C, Leung S, Bowen NJ, Ionescu DN, Rajput A, Prentice LM, Miller D, Santos J, Swenerton K, Gilks CB, Huntsman D (2008) Ovarian carcinoma subtypes are different diseases: implications for biomarker studies. PLoS Med 5(12): e232

Konstantinopoulos PA, Cannistra SA, Fountzilas H, Culhane A, Pillay K, Rueda B, Cramer D, Seiden M, Birrer M, Coukos G, Zhang L, Quackenbush J, Spentzos D (2011) Integrated analysis of multiple microarray datasets identifies a reproducible survival predictor in ovarian cancer. PLoS One 6(3): e18202

Kristensen GB, Trope C (1997) Epithelial ovarian carcinoma. Lancet 349(9045): 113-117

Langdon SP, Lawrie SS, Hay FG, Hawkes MM, McDonald A, Hayward IP, Schol DJ, Hilgers J, Leonard RC, Smyth JF (1988) Characterization and properties of nine human ovarian adenocarcinoma cell lines. Cancer Res 48(21): 6166-6172

Ledermann JA, Raja FA (2010) Targeted trials in ovarian cancer. Gynecol Oncol 119(1): 151-156

Lee KR, Young RH (2003) The distinction between primary and metastatic mucinous carcinomas of the ovary: gross and histologic findings in 50 cases. Am J Surg Pathol 27(3): 281-292

Lee Y, Miron A, Drapkin R, Nucci MR, Medeiros F, Saleemuddin A, Garber J, Birch C, Mou H, Gordon RW, Cramer DW, McKeon FD, Crum CP (2007) A candidate precursor to serous carcinoma that originates in the distal fallopian tube. J Pathol 211(1): 26-35

Lu Y, Han J, Scanlon KJ (1988) Biochemical and molecular properties of cisplatin-resistant A2780 cells grown in folinic acid. J Biol Chem 263(10): 4891-4894

Lynch TJ, Bell DW, Sordella R, Gurubhagavatula S, Okimoto RA, Brannigan BW, Harris PL, Haserlat SM, Supko JG, Haluska FG, Louis DN, Christiani DC, Settleman J, Haber DA (2004) Activating mutations in the epidermal growth factor receptor underlying responsiveness of non-small-cell lung cancer to gefitinib. $N$ Engl J Med 350(21): 2129-2139

Matei D, Emerson RE, Lai YC, Baldridge LA, Rao J, Yiannoutsos C, Donner DD (2006) Autocrine activation of PDGFRalpha promotes the progression of ovarian cancer. Oncogene 25(14): 2060-2069

Mi RR, Ni H (2003) MDM2 sensitizes a human ovarian cancer cell line. Gynecol Oncol 90(2): 238-244

Mosmann T (1983) Rapid colorimetric assay for cellular growth and survival: application to proliferation and cytotoxicity assays. J Immunol Methods 65(1-2): 55-63

Ohishi Y, Oda Y, Uchiumi T, Kobayashi H, Hirakawa T, Miyamoto S, Kinukawa N, Nakano H, Kuwano M, Tsuneyoshi M (2002) ATPbinding cassette superfamily transporter gene expression in human primary ovarian carcinoma. Clin Cancer Res 8(12): 3767-3775

Peklak-Scott C, Smitherman PK, Townsend AJ, Morrow CS (2008) Role of glutathione S-transferase P1-1 in the cellular detoxification of cisplatin. Mol Cancer Ther 7(10): 3247-3255 
Perren TJ, Swart AM, Pfisterer J, Ledermann JA, Pujade-Lauraine E, Kristensen G, Carey MS, Beale P, Cervantes A, Kurzeder C, du Bois A, Sehouli J, Kimmig R, Stahle A, Collinson F, Essapen S, Gourley C, Lortholary A, Selle F, Mirza MR, Leminen A, Plante M, Stark D, Qian W, Parmar MK, Oza AM (2011) A phase 3 trial of bevacizumab in ovarian cancer. N Engl J Med 365(26): 2484-2496

Pliarchopoulou K, Pectasides D (2011) Epithelial ovarian cancer: focus on targeted therapy. Crit Rev Oncol Hematol 79(1): 17-23

Prakash J, de Jong E, Post E, Gouw AS, Beljaars L, Poelstra K (2010) A novel approach to deliver anticancer drugs to key cell types in tumors using a PDGF receptor-binding cyclic peptide containing carrier. J Control Release 145(2): 91-101

Scottish Intercollegiate Guidelines Network Epithelial Ovarian Cancer: A National Clinical Guideline (2003) ISBN 1899893938

Smith G, Dawe RS, Clark C, Evans AT, Comrie MM, Wolf CR, Ferguson J, Ibbotson SH (2003a) Quantitative real-time reverse transcriptionpolymerase chain reaction analysis of drug metabolizing and cytoprotective genes in psoriasis and regulation by ultraviolet radiation. $J$ Invest Dermatol 121(2): 390-398

Smith G, Wolf CR, Deeni YY, Dawe RS, Evans AT, Comrie MM, Ferguson J, Ibbotson SH (2003b) Cutaneous expression of cytochrome P450 CYP2S1: individuality in regulation by therapeutic agents for psoriasis and other skin diseases. Lancet 361(9366): 1336-1343
Tothill RW, Tinker AV, George J, Brown R, Fox SB, Lade S, Johnson DS, Trivett MK, Etemadmoghadam D, Locandro B, Traficante $N$ Fereday S, Hung JA, Chiew YE, Haviv I, Gertig D, DeFazio A, Bowtell DD (2008) Novel molecular subtypes of serous and endometrioid ovarian cancer linked to clinical outcome. Clin Cancer Res 14(16): 5198-5208

Townsend DM, Tew KD (2003) The role of glutathione-S-transferase in anti-cancer drug resistance. Oncogene 22(47): 7369-7375

Turner N, Grose R (2010) Fibroblast growth factor signalling: from development to cancer. Nat Rev Cancer 10(2): 116-129

Wiegand KC, Shah SP, Al-Agha OM, Zhao Y, Tse K, Zeng T, Senz J, McConechy MK, Anglesio MS, Kalloger SE, Yang W, Heravi-Moussavi A, Giuliany R, Chow C, Fee J, Zayed A, Prentice L, Melnyk N, Turashvili G, Delaney AD, Madore J, Yip S, McPherson AW, Ha G, Bell L, Fereday S, Tam A, Galletta L, Tonin PN, Provencher D, Miller D, Jones SJ, Moore RA, Morin GB, Oloumi A, Boyd N, Aparicio SA, Shih Ie M, Mes-Masson AM, Bowtell DD, Hirst M, Gilks B, Marra MA, Huntsman DG (2010) ARID1A mutations in endometriosis-associated ovarian carcinomas N Engl J Med 363(16): 1532-1543

Zorn KK, Bonome T, Gangi L, Chandramouli GV, Awtrey CS, Gardner GJ, Barrett JC, Boyd J, Birrer MJ (2005) Gene expression profiles of serous, endometrioid, and clear cell subtypes of ovarian and endometrial cancer. Clin Cancer Res 11(18): 6422-6430

This work is published under the standard license to publish agreement. After 12 months the work will become freely available and the license terms will switch to a Creative Commons Attribution-NonCommercial-Share Alike 3.0 Unported License. 